\title{
Comparison of online single-breath vs. online multiple-breath exhaled nitric oxide in school-age children
}

\author{
Oliver Fuchs ${ }^{1}$, Philipp Latzin ${ }^{1}$, Florian Singer ${ }^{1}$, Nicole Petrus ${ }^{2}$, Elena Proietti ${ }^{1}$, Elisabeth Kieninger ${ }^{1}$, Carmen Casaulta ${ }^{1}$ \\ and Urs Frey ${ }^{3}$
}

INTRODUCTION: Standards for online multiple-breath (mb) exhaled nitric oxide (eNO) measurements and studies comparing them with online single-breath (sb) eNO measurements are lacking, although eNOmb requires less cooperation in children at school age or younger.

METHODS: Online eNOmb and eNOsb were measured in 99 healthy children and (in order to observe higher values) in 21 children with suspected asthma at a median age of 6.1 and $11.7 \mathrm{y}$, respectively. For eNOmb, we aimed for 20 tidal breathing maneuvers; eNOsb was measured according to standards. The two techniques were compared by standard methods after computing $\mathrm{NO}$ output or extrapolating eNOmb to the standard flow of $50 \mathrm{ml} / \mathrm{s}\left(\mathrm{eNOmb} \mathrm{m}_{50}\right.$ ).

RESULTS: Measurements were acceptable in 82 (eNOmb) and 81 (eNOsb) children. Paired data were available for 65 children. On a log-log scale, eNOmb ${ }_{50}$ (geometric mean \pm SD $13.1 \pm$ 15.5 parts per billion, ppb) was correlated with eNOsb (12.5 \pm $15.8 \mathrm{ppb}$ ), with $r^{2}=0.87$. The mean difference between eNOsb and eNOmb 50 was $-0.7 \mathrm{ppb}$, with limits of agreement (LOAs) of 4.0 and $-5.3 \mathrm{ppb}$.

DISCUSSION: Despite its correlation with eNOsb, the LOA range hampers eNOmb use in research, where exact values across the whole range are warranted. However, eNOmb might be an alternative tool especially at preschool age, when cooperation during measurements is crucial.

hildhood asthma resembles a complex syndrome and is of major public health relevance because of its high prevalence (1-3). Exhaled nitric oxide (eNO) serves as a surrogate measure of allergic airway inflammation. It is correlated with the expression of inducible NO synthase in airways of atopics, especially individuals with atopic asthma $(4,5)$. In both the clinical and the research settings, eNO aids in discriminating different asthma phenotypes, particularly the atopic vs. the nonatopic form $(6,7)$. Increased in atopic or asthmatic patients and reduced under corticosteroid treatment, eNO has assisted with diagnosis and clinical guidance for pediatric and adult patients alike (8).

The single-breath online method for eNO measurement (eNOsb) is the gold standard for older children, adolescents, and adults according to guidelines by the European Respiratory Society (ERS) and the American Thoracic Society (ATS; ref. 9). Standardization is necessary for the collection of acceptable and comparable data both within one center and between several centers. For eNOsb measurements, standardization primarily involves the expiratory target flow of $50 \mathrm{ml} / \mathrm{s}$, as eNO is inversely related to flow (9-13). Furthermore, standardization requires the following criteria: inhalation to total lung capacity during the inspiratory maneuver, inspired $\mathrm{NO}$ concentration of $<5$ parts per billion (ppb), exhalation time of $\geq 4 \mathrm{~s}$ for children $<12$ y and $\geq 6 \mathrm{~s}$ if older, duration of expiratory plateau at target flow of $\geq 2 \mathrm{~s}$, expiratory pressure level of $5-20 \mathrm{~cm} \mathrm{H}_{2} \mathrm{O}$ for velum closure, calibration, hardware, and measurement techniques (9). Performing tests successfully according to guidelines is strongly related to age $(9,14,15)$. Children often find it demanding to perform acceptable measurements, especially at preschool age. Lack of cooperation and a relatively short attention span further complicate matters (9). A method based on quiet tidal breathing might be easier to perform satisfactorily. This is comparable to pulmonary function tests at this age. Here, measurements based on quiet tidal breathing usually result in higher proportions of children being able to perform the maneuver satisfactorily than spirometry as a forced expiratory maneuver, as illustrated in a review on lung function tests in preschool children with cystic fibrosis (16). Until now, multiple-breath eNO (eNOmb) measurements have mostly been performed in infants and toddlers (17-21). Despite requiring less cooperation beyond infancy, criteria for standardization of eNOmb measurements are not yet included in the current ERS/ATS guidelines due to methodological issues (9). Omitting the need for flow control, it may be possible to overcome this difficult hurdle during eNO measurements according to ERS/ATS standards in young children.

Within this article, we hypothesized that eNOmb might constitute an alternative to the current gold standard of eNOsb measurements at school age and set out to close the gap of missing evidence for (i) feasibility and quality control, (ii) correlation, and (iii) accuracy of eNOmb in comparison with eNOsb measurements. We did so in study participants

'Department of Pediatrics, Inselspital, University of Bern, Bern, Switzerland; ${ }^{2}$ Department of Pediatric Respiratory Medicine and Allergy, Emma Children's Hospital Academic Medical Center, University of Amsterdam, Amsterdam, The Netherlands; ${ }^{3}$ University Children's Hospital, University of Basel, Basel, Switzerland. Correspondence: Urs Frey (urs.frey@ukbb.ch) 
of the Bern Infant Lung Development (BILD) cohort $(20,22)$ who were enrolled in follow-up at the age of 5-6 y and, to also assess comparability at higher eNO values, in children around $11 \mathrm{y}$ of age with suspected asthma presenting for routine workup in the asthma outpatient clinic of the University Children's Hospital in Bern, Switzerland.

\section{RESULTS}

Feasibility and Quality Control of eNOmb Measurements as Compared With the Gold Standard of eNOsb Measurements

Online eNOmb and eNOsb measurements were performed in 120 children; 99 were BILD cohort study participants and 21 were children with suspected asthma. Table 1 displays demographic characteristics and results of eNO measurements separately for cohort study participants and subjects with suspected asthma.

Figure 1 presents numbers of acceptable eNO measurements in the whole study population as well as separately for BILD cohort study participants and subjects with suspected asthma. Numbers of eNO measurements excluded from analyses due to quality control and ATS/ERS standards are also displayed (9). We obtained acceptable eNOmb measurements and eNOsb measurements in the whole study population in $82(68.3 \%)$ and $81(67.5 \%)$ children, respectively $(P=0.89)$. Among those with acceptable eNOmb measurements, 17 (20.7\%) were unable to perform acceptable eNOsb measurements $(n=14$ BILD cohort study participants). Among those able to perform eNOsb measurements according to standards, 16 (19.8\%) were not able to acceptably perform eNOmb measurements $(n=13$ BILD cohort study participants). For BILD cohort participants, we found a trend toward a higher proportion of boys $(P=0.07)$ and toward higher body weight $(P=0.10)$ among children unable to perform acceptable eNOsb measurements. The trend for a higher proportion of boys among BILD cohort study participants was also found for children not able to produce acceptable pairs of eNOmb and eNOsb measurements $(P=0.09)$. There were no other differences between children who were able or unable to perform each measurement in an acceptable way, or children who were deemed to have pairs of acceptable tests.
Correlation of eNOmb Measurements With the Gold Standard of eNOsb Measurements

To compare the two techniques, we analyzed data pairs with both acceptable eNOmb and eNOsb measurements. Table 2 displays geometric means (SD) for ln-transformed results of acceptable $\mathrm{eNOmb}_{50}$ (eNOmb extrapolated to the standard flow of $50 \mathrm{ml} / \mathrm{s}$ ) and eNOsb, as well as of NO output derived from multiple-breath measurements ( $\left.\mathrm{V}^{\prime} \mathrm{NOmb}\right)$ and $\mathrm{NO}$ output derived from single-breath measurements ( $\mathrm{V}^{\prime} \mathrm{NOsb}$ ) together with their respective intra- and between-measurement coefficients of variation. Both on a $\ln -\ln$ and a $\log -\log$ scale, online $\mathrm{eNOmb}_{50}$ values were correlated with results of eNOsb measurements. As displayed in Figure 2a, this correlation was significant. The same significant correlation was found when we analyzed $\mathrm{V}^{\prime} \mathrm{NOmb}$ compared with $\mathrm{V}^{\prime} \mathrm{NOsb}$ (Figure $2 \mathbf{b}$ ). In contrast to eNOmb ${ }_{50}$, correlation of raw eNOmb results with eNOsb results on either a $\ln -\ln$ or log-log scale was inferior (slope $0.75, P<0.0001, r^{2}=0.67$ ).

\section{Accuracy of eNOmb Measurements as Compared With the Gold Standard of eNOsb Measurements}

In a last analysis, we wanted to assess the accuracy of eNOmb as compared with the gold standard of eNOsb measurements. Figure 3a,b displays Bland-Altman plots for this comparison and of $\mathrm{V}^{\prime} \mathrm{NOmb}$ with $\mathrm{V}^{\prime} \mathrm{NOsb}$, correspondingly. As differences between the two methods were normally distributed, these are presented as raw data on a linear scale. Means of either eNO or $\mathrm{V}^{\prime} \mathrm{NO}$ values were right-skewed and means of paired measurements are thus presented on a log-scale, however, without data transformation.

We found that $\mathrm{eNOmb}_{50}$ measurements systemically resulted in higher values as compared with eNOsb measurements with upper and lower limits of agreement (LOAs) of 4.0 and $-5.3 \mathrm{ppb}$, respectively, and a total mean difference of $-0.7 \mathrm{ppb}$ across the whole data range. If means of paired eNO measurements were $>20.0 \mathrm{ppb}(n=11)$, these numbers were 6.4 and $-5.0 \mathrm{ppb}$, respectively, with a total mean difference of $0.7 \mathrm{ppb}$, and if restricted to means of paired eNO measurements of $\leq 20 \mathrm{ppb}(n=54)$, these numbers were 3.3 and $-5.1 \mathrm{ppb}$, respectively, with a total mean difference of $0.9 \mathrm{ppb}$. Also $\mathrm{V}^{\prime} \mathrm{NOmb}$ measurements resulted in

Table 1. Demographic characteristics and eNO results of the whole study population

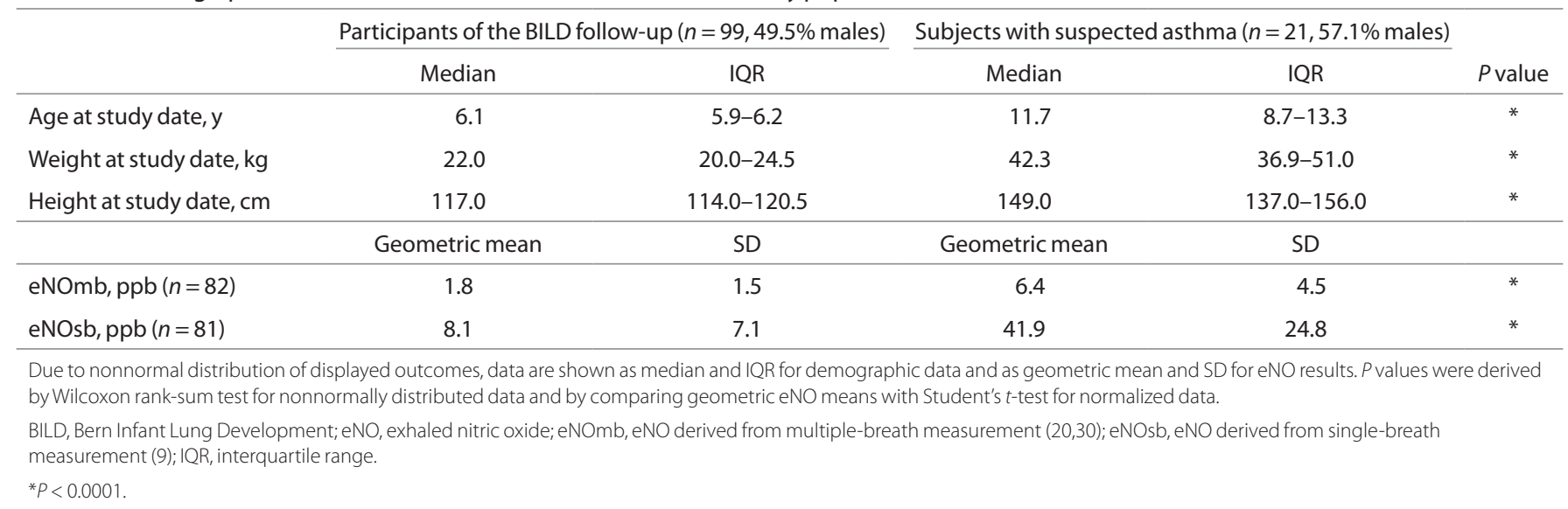




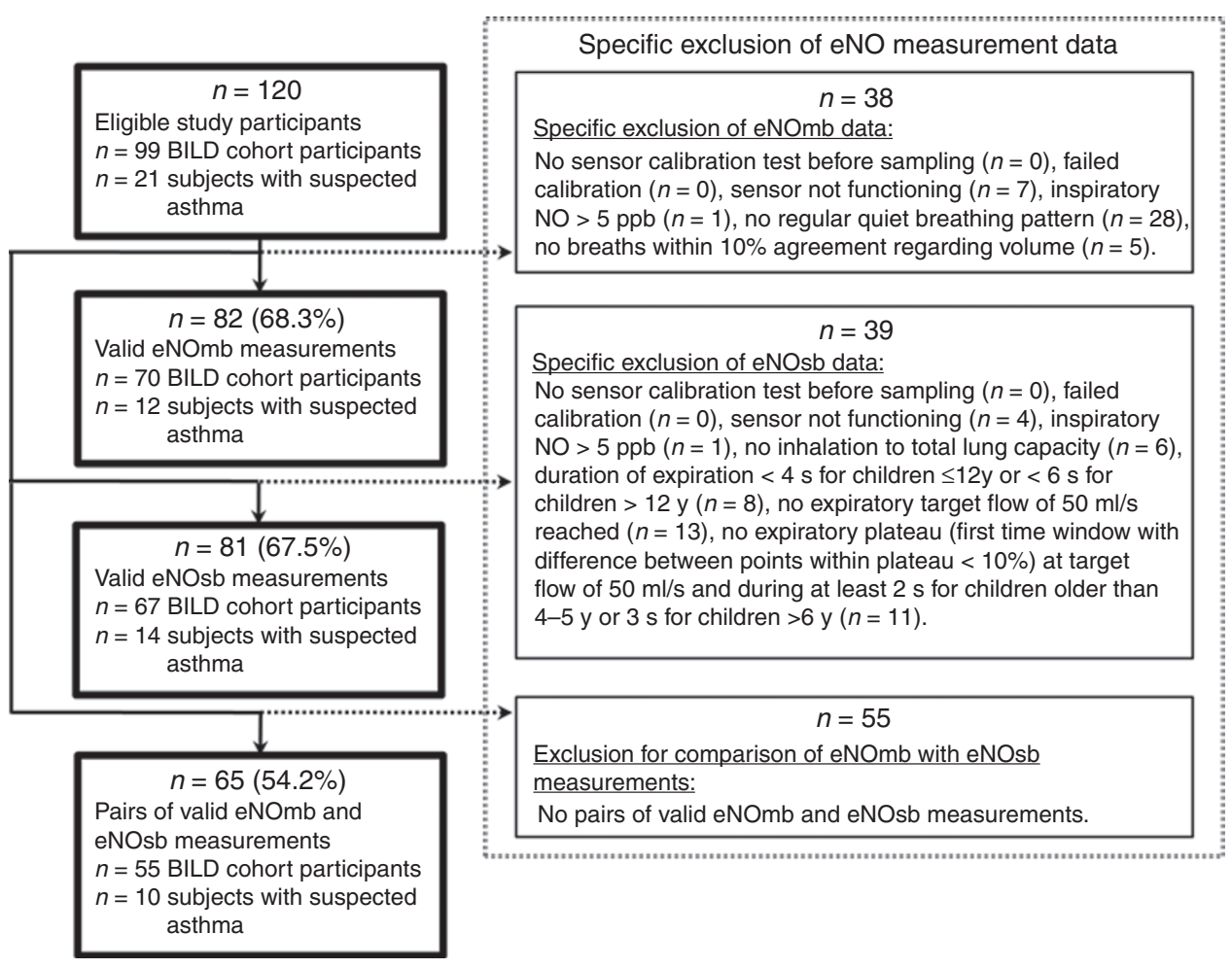

Figure 1. Numbers of acceptable eNO measurements and of data sets excluded from analyses. The figure displays numbers (proportion) of acceptable eNOmb and eNOsb measurements in the whole study population and per BILD cohort participants and subjects with suspected asthma on the left. Numbers of individual measurements excluded from analyses are shown on the right together with the respective criteria. Specific numbers for each exclusion criterion are given in parentheses; due to overlap they can add up to numbers higher than total number excluded. BILD, Bern Infant Lung Development; eNO, exhaled nitric oxide; eNOmb, eNO derived from multiple-breath measurement; eNOsb, eNO derived from single-breath measurement; ppb, parts per billion.

Table 2. Results for pairs of acceptable eNOmb and eNOsb measurements $(n=65)$

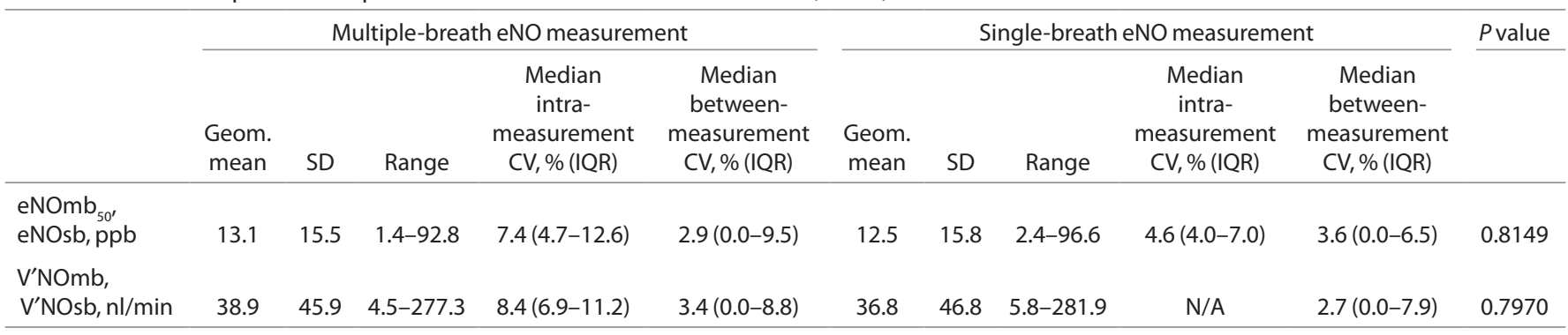

Due to nonnormal distribution of displayed outcomes, data are shown as geometric mean and SD of In-transformed data. $P$ values were derived by comparing geometric means with Student's $t$-test after their distributions were normalized.

CV, coefficient of variation; eNO, exhaled nitric oxide; eNOmb, eNO derived from multiple-breath measurement (20,30); eNOmb ${ }_{50}$ eNO derived from multiple-breath measurement $(20,30)$ and additionally extrapolated to flow of $50 \mathrm{ml} / \mathrm{s}$; eNOsb, eNO derived from single-breath measurement (9); Geom., geometric; IQR, interquartile range; N/A, not applicable; $V^{\prime} N O m b$, NO output derived from multiple-breath measurement (20,30); $V^{\prime} N O s b$, NO output derived from single-breath measurement (9).

systemically higher values as compared with $\mathrm{V}^{\prime} \mathrm{NOsb}$ measurements. Across the whole data range, upper and lower LOAs were 12.8 and $-17.0 \mathrm{nl} / \mathrm{s}$ with a total mean difference of $-2.1 \mathrm{nl} / \mathrm{s}$. If restricted to means of paired eNO measurements $>20.0 \mathrm{ppb}$, these numbers were 21.7 and $-16.0 \mathrm{nl} / \mathrm{s}$ with a total mean difference of $2.8 \mathrm{nl} / \mathrm{s}$, and if means of paired eNO measurements were $\leq 20.0 \mathrm{ppb}$, these numbers were 10.2 and $-16.4 \mathrm{nl} / \mathrm{s}$ with a total mean difference of $-3.1 \mathrm{nl} / \mathrm{s}$, respectively.

Upper and lower LOAs for the ratios of difference of paired eNO measurements over mean of paired eNO measurements for the whole data range were $52.7 \%$ and $-65.4 \%$, with a total mean of $-6.3 \%$. Restricted to means of paired eNO measurements $>20.0 \mathrm{ppb}$, these numbers were $23.4 \%$ and $-16.7 \%$ with a mean of $3.3 \%$, and if means of paired eNO measurements were $\leq 20.0 \mathrm{ppb}$, these numbers were $55.3 \%$ and $-71.9 \%$ with a mean of $-8.3 \%$.

\section{DISCUSSION}

In summary, we found that eNOmb measurements are at least as feasible as eNOsb measurements with all data being collected under strict quality control and the latter measured according to currently available ERS/ATS standards (9). 

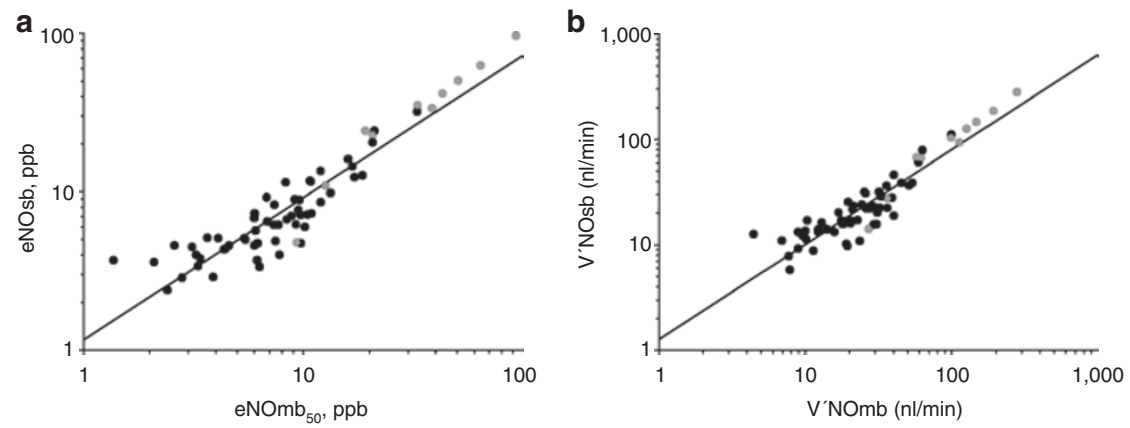

Figure 2. Correlation between different eNO measurement techniques. The figure displays the correlation (a) between eNOsb and eNOmb ${ }_{50}($ slope 0.90 , $r^{2}=0.87, P<0.0001$ ) and (b) between $V^{\prime} N O s b$ and $V^{\prime} N O m b$ (slope $0.90, r^{2}=0.857, P<0.0001$ ) on a log-log scale. In both a and $\mathbf{b}$, gray dots indicate results from subjects with suspected asthma, black dots indicate results from participants of the BILD cohort, and lines represent regression lines. eNO, exhaled nitric oxide; eNOmb, eNO derived from multiple-breath measurement; eNOmb ${ }_{50}$ eNOmb additionally extrapolated to flow of $50 \mathrm{ml} / \mathrm{s}$; eNOsb, eNO derived from single-breath measurement; ppb, parts per billion; $\mathrm{V}^{\prime} \mathrm{NOmb}$, NO output derived from multiple-breath measurement; $\mathrm{V}^{\prime} \mathrm{NO}$ sb, NO output derived from single-breath measurement.
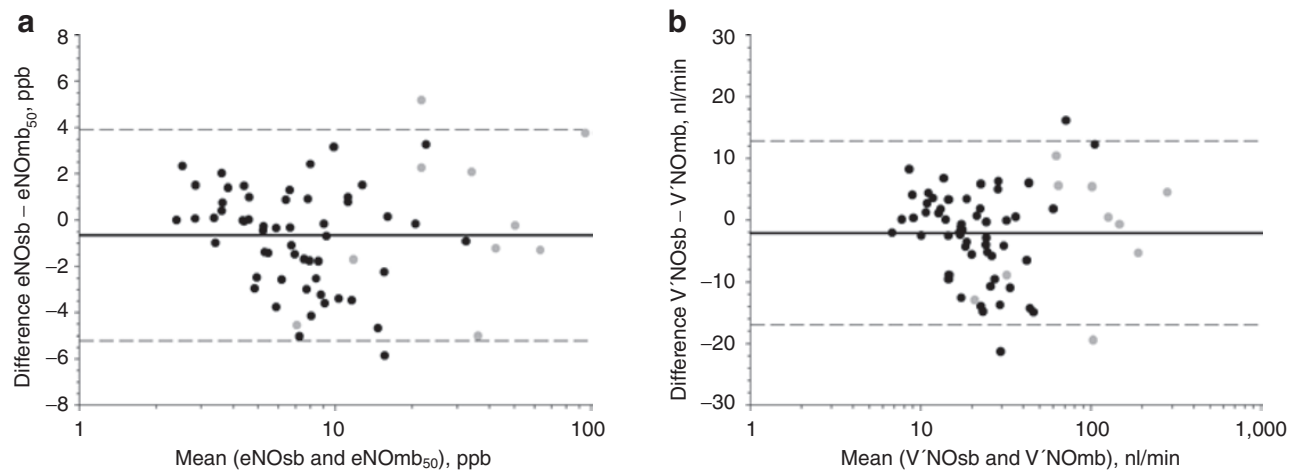

Figure 3. Bland-Altman plots of different eNO measurement techniques. The figure displays Bland-Altman plots, (a) means of eNOmb ${ }_{50}$ and eNOsb per measurement on the $x$ axis (log-scale) plotted against the differences of eNOsb-eNOmb ${ }_{50}$ per measurement on the $y$ axis, and (b) means of $V^{\prime} N O m b$ and $V^{\prime} N O s b$ per measurement on the $x$ axis (log-scale) plotted against the differences of V'NOsb-V'NOmb per measurement on the $y$ axis. In both $\mathbf{a}$ and $\mathbf{b}$, solid thick lines represent mean difference, dashed lines represent upper (mean difference + 1.96 SD of differences) and lower (mean difference - 1.96 SD of differences) limits of agreement, gray dots indicate results from subjects with suspected asthma, and black dots indicate results from participants of the BILD cohort. eNO, exhaled nitric oxide; eNOmb, eNO derived from multiple-breath measurement; eNOmb ${ }_{50}$, eNOmb additionally extrapolated to flow of $50 \mathrm{ml} / \mathrm{s}$; eNOsb, eNO derived from single-breath measurement; ppb, parts per billion; $\mathrm{V}^{\prime} \mathrm{NOmb}$, NO output derived from multiple-breath measurement; V'NOsb, NO output derived from single-breath measurement.

Results from eNOmb measurements were highly correlated with the gold standard within the range of assessed eNO values. Furthermore, irrespective of resulting in slightly higher eNO values and some outliers, eNOmb measurements demonstrated agreement with measurements using the single-breath technique. Thus, eNOmb measurements may constitute an alternative to the more difficult single-breath measurements at school age or around school entry, and especially if children are unable to perform eNOsb measurements according to current ERS/ATS guidelines due to a lack of cooperation.

The method used to measure eNOmb in this study is a tidal breathing technique with uncontrolled flow rate, thus not requiring continuous adaptation of exhalation resistance or automatic flow rate control. As yet, no standardized tidal breathing method to measure eNO in children exists that is recommended in current guidelines for the clinical setting (9). Our aim was to offer an alternative to eNOsb measurements, which are often difficult to perform within the acceptability criteria, especially in preschool children (9). Age-related difficulties were documented in several studies, such as by Napier et al. regarding eNO-related methodological issues in 61 children aged $4-6$ y, younger than our study participants (15). These were further demonstrated in a study by Ito et al. in 165 asthmatic children, who, with a median age of $7 y$, were older than the majority of our study population (14). Here, study participants who were naive with regard to eNO measurements displayed age-related success rates: those younger than $8 \mathrm{y}$ were less successful than older children (14).

For lung function tests, acceptable performance is age-dependent and particularly low at preschool age. This is particularly the case if one compares proportions of acceptable data derived from spirometry with those from other pulmonary function tests based on quiet tidal breathing maneuvers. This is illustrated in a review of lung function tests in children with cystic fibrosis at preschool age and also depicted in the current ATS/ERS guidelines for pulmonary function testing in preschool children $(16,23)$. As compared with techniques not based on forced expiratory maneuvers, the feasibility of spirometry is lowest for this age group, with the majority of studies demonstrating success rates of $<50 \%$ 
for children aged $2 \mathrm{y}$ and $\geq 50 \%$ for older children. In contrast, measurements based on tidal breathing (such as the forcedoscillation technique or multiple-breath washouts) revealed higher success rates of $\geq 80 \%$ after the age of $4 \mathrm{y}$ (16). Hence, simplifying measurements is important for the clinical setting; however, it is necessary to do so without losing value in the diagnostic process. This underlines the importance and the need for easy-to-perform techniques in the age group of young children.

The single-breath method to measure eNO levels is well established for asthma but also for diseases other than asthma and in research $(24,25)$. In this context, different devices for eNOsb measurements have been compared, also with regard to their applicability. Kalliola et al. compared the use of a portable device for eNOsb measurements to a stationary device in 55 children with a mean age of 5.7 y (26). That study revealed poor accuracy at low eNO levels and lower success rates $(73 \%)$ for the portable device as compared with the stationary device (93\%; ref. 26). Their proportion of successfully performed eNOsb measurements among children with a mean age comparable to the majority of our study population, the BILD cohort study participants, was generally higher than ours (67.5\% for eNOsb). However, it remains unclear whether study participants in the study by Kalliola et al. were also naive to eNO measurements. Furthermore, their study was designed to compare two devices for eNOsb measurements. The already mentioned study by Ito et al. has compared different exhalation times ( $6 \mathrm{~s}$ vs. $10 \mathrm{~s}$ ) for eNOsb measurements with the same portable device, with a duration of $6 \mathrm{~s}$ demonstrating better feasibility in children $<8$ y $(92.0 \%$ vs. $60 \%)$ while still in accordance with guidelines (14). Their proportion of acceptable measurements in children naive to eNO measurements was again higher than ours; however, in the study by Ito et al. it remains unclear whether study participants were also naive to spirometry. There are studies that, similar to ours, compare the gold standard with a potentially easier eNO measurement technique $(18,27)$. Buchvald et al. sampled eNO using an online tidal breathing method at a fixed flow rate (controlled eNO) and in mixed expired air in 16 patients with asthma $7-14 \mathrm{y}$ old and 51 preschoolers (14 healthy, age 2-5 y) and compared these techniques with eNOsb among school-age children only. They could show that in preschoolers controlled eNO measurement was feasible and showed better agreement with eNOsb measurement than eNO measurement in mixed expired air among schoolchildren. However, the tidal breathing technique was different, the children in whom techniques were directly compared were older than ours, and it is unlikely that they were naive to eNO measurements or spirometry (18). Hadjikoumi et al. sampled eNO in mixed expired air in 25 healthy children, mean age $11.5 \mathrm{y}$, and 20 asthmatics, mean age $12 \mathrm{y}$, thus older than our study population (27). Success rates were not reported, neither technique demonstrated good correlation, and disease groups were less correctly identified by eNO in mixed expired air as compared with eNOsb measurements (27).
To the best of our knowledge, this is the first study that compared and assessed feasibility as well as quality control, correlation, and accuracy of online eNOmb with uncontrolled flow rate with the current gold standard at school age. For this, we used a very simple method to linearly extrapolate eNOmb results to the recommended target flow for eNOsb measurements. All data were collected in a setup with a number of methodological strengths. Measurements were always performed in the same order and with the same equipment. Thus, the identical careful approach for regular calibration was used (9). Furthermore, we also applied extensive quality control during measurements and during later analyses for comparison of the two techniques. Our eNOsb measurements completely adhere to the latest ATS/ERS standards (9). To compare the simpler eNOmb method with the gold standard, we adopted a conservative approach to assessing its acceptability. Furthermore, all study participants performed eNO measurements for the first time and were also naive with regard to spirometry.

With participants of the population-based BILD cohort study as the majority of the study population, the study may be biased toward a well-educated middle-class population (22). The children who were seen for the first time in our asthma outpatient clinic were included to also cover higher eNO levels in our analyses. They reflect a pediatric population referred to a major center for work-up of suspected asthma and were older than the BILD cohort study participants. However, the focus of our study was not to assess differences in feasibility of the two measurement techniques comparing healthy and children with suspected asthma or comparing different age groups or to assess factors influencing feasibility of assessed measurement techniques. For this, the study would have been underpowered anyway. Our aim was to compare two eNO measurement techniques with regard to their feasibility in direct comparison to each other, to assess their correlation, and the accuracy in relation to the current gold standard. The limitations of our study have to be kept in mind before our findings can be extrapolated to other populations. Furthermore, the simple linear method to extrapolate eNOmb results to the recommended eNOsb target flow may be an oversimplification of the complex mechanisms underlying different eNO levels in health and disease. More elaborate methods that account for the complexity upstream to what we measure as eNO have been published (10). As eNO is flow dependent in a nonlinear fashion, these models aim at modeling NO production in the lung and shedding light on factors influencing NO production dependent and independent of flow $(10,13,28)$. However, they lack the simplicity and ease of use of the multiple-breath method with uncontrolled flow rate, especially for young children.

\section{Conclusion}

If online eNOmb measurements are performed adhering to strict quality control, they seem to be as feasible as the current gold standard of eNOsb measurements and to produce similar results. Despite their significant correlation, the wide range of LOAs and systematically slightly higher levels of eNOmb in 
comparison to eNOsb measurements hampers eNOmb use in research, where exact values across the whole range are warranted. Furthermore, other techniques such as measurements of the fraction of eNO in exhaled air collected in NO-inert bags (offline fraction of eNO measurement) have been shown to be suitable for use in research and clinical use, especially for infants (29). Multiple-breath eNO measurements are less dependent on cooperation and might constitute a promising alternative, particularly for younger children at preschool age, when lack of cooperation represents a crucial problem, especially in the clinical setting. Differences in eNO levels for discrimination between disease groups are greater by far than the differences between the different measurement techniques in our study, especially at higher eNO levels. However, before eNOmb measurements may be used in daily clinical practice or as an alternative technique at preschool age, further testing is required.

\section{METHODS}

\section{Study Population}

Data were primarily collected in participants during follow-up of an ongoing prospective birth cohort of unselected, healthy subjects, the BILD cohort (22). For initial recruitment, the following inclusion criteria were applied: white ethnicity; term delivery; no severe maternal health problems; no maternal drug abuse other than nicotine; and no known major birth defects or perinatal disease of the newborn, such as respiratory distress, airway malformation, or other major respiratory diseases diagnosed after birth (22). To include higher eNO values in the analyses, subjects attending the pulmonary outpatient clinic of the University Children's Hospital of Bern, Switzerland, for the first time due to suspected asthma were additionally recruited. Children were eligible only if they had never performed spirometry or either of the two eNO measurement techniques (eNOsb and eNOmb) before, except for the infant lung function measurements of participants of the BILD cohort (20). This was ascertained before the measurement by personal interview. Otherwise, exclusion criteria for eNO measurements according to ERS/ATS standards applied (9).

The study was approved by both the Ethics Committee of the Canton of Bern, Switzerland (Kantonale Ethikkommission Bern), and the Research Ethics Committee of University Hospital Bern, Switzerland (Inselspital). All caregivers provided written informed consent for this study.

\section{NO Measurements}

Measurements were always performed in the same order: we aimed for three eNOmb measurements followed by three acceptable eNOsb measurements. For both online eNOsb and eNOmb methods, eNO was measured with a rapid-response chemoluminescence analyzer (CLD 88 sp; EcoMedics, Duernten, Switzerland). Flow was recorded using an ultrasonic flow meter (Spiroson; EcoMedics). For signal collection, a software package (WBreath 3.28; ndd Medical Technologies, Zurich, Switzerland) was used and tidal flows and derived volumes were converted to body temperature and ambient pressure, and saturated with water vapor conditions. We measured eNOmb breath by breath during the third quartile of expiration and calculated mean eNO, correcting for expiratory flow $\left(\mathrm{V}^{\prime} \mathrm{E}\right)$ without flow control as described previously $(20,30)$. For both eNOmb and eNOsb measurements, main outcome parameters were eNO and $\mathrm{NO}$ output $\left(\mathrm{V}^{\prime} \mathrm{NO}\right.$, eNO concentration multiplied by corresponding expiratory flow, $\left.V^{\prime} \mathrm{E}\right)$. Results for eNOmb were extrapolated to a flow of $50 \mathrm{ml} / \mathrm{s}$ $\left(\mathrm{eNOmb}_{50}\right)$ to allow comparison with the gold standard using the following formula (rule of proportions):

$$
\mathrm{eNOmb}_{50}(\mathrm{ppb})=\mathrm{eNOmb}(\mathrm{ppb}) \times\left(V^{\prime} E(\mathrm{ml} / \mathrm{s}) / 50 \mathrm{ml} / \mathrm{s}\right)
$$

\section{Quality Control of NO Measurements}

(i) We performed sensor calibration tests before each measurement. (ii) Contamination by ambient NO was avoided by always using NO-free air for inspiration in the case of both measurement techniques. (iii) For eNOmb measurement, we aimed for 20 quiet tidal breathing maneuvers. (iv) From each of the multiple-breath measurements, mean eNOmb measurement was calculated only from breaths that were within $10 \%$ agreement regarding volume. (v) Total mean eNOmb value was then calculated from only two to three measurements that agreed within $10 \%$ or from two within $5 \%$ (ref. 9). Online eNOsb was measured and calculated according to current ATS/ERS standards (9).

\section{Statistical Analyses}

Due to the eNO data being right-skewed, we ln-transformed results to normalize their distribution and calculated geometric means and their differences. We measured the correlation of eNOsb with $\mathrm{eNOmb}_{50}$ as well as of $\mathrm{V}^{\prime} \mathrm{NOsb}$ with $\mathrm{V}^{\prime} \mathrm{NOmb}$ by linear regression analyses to calculate $r^{2}$ for log-transformed data to normalize their distribution. Results were identical after ln-transformation. Means of normally distributed data were compared by $t$-test, proportions were compared by the binomial test of proportion, and the Wilcoxon ranksum test was used for nonnormally distributed data. A $P$ value $<0.05$ was considered significant.

We calculated intra- and between-measurement coefficients of variation (\%). Intra-measurement coefficients of variation for eNOmb were computed both for eNO and $\mathrm{V}^{\prime} \mathrm{NO}$ as the ratio of the standard deviation of acceptable breaths over the mean of the whole measurement. Intra-measurement coefficients of variation for eNOsb could be calculated for eNO only as the standard deviation over the mean eNO during the expiratory plateau at target flow.

Accuracy of raw results from eNOmb ${ }_{50}$ as compared with eNOsb was determined using the Bland-Altman method by plotting differences of paired measurements against means of paired measurements and by calculating upper and lower LOAs as mean difference of paired measurements \pm SD of difference of paired measurements (31). We further calculated upper and lower LOAs for the ratios of difference of paired measurements over mean of paired measurements (\%). These analyses were performed over the whole range of values and additionally for means of paired eNO measurements $>20.0$ and $\leq 20.0 \mathrm{ppb}$, separately. Descriptive statistics and linear regression analyses were performed using STATA 11 for Windows (STATA Corporation, College Station, TX).

\section{ACKNOWLEDGMENTS}

We thank all patients, study participants, and families for their participation. Furthermore, we thank the study nurses Monika Graf, Barbara Hofer, Sandra Luescher, and Christine Becher, and lung function technicians Gisela Wirz and Sharon Schmid for their invaluable assistance and support, as well as Janet Maccora for proofreading of the manuscript.

\section{STATEMENT OF FINANCIAL SUPPORT}

This work was supported by the Swiss National Science Foundation (grant no. 3200-B0-12099 to O.F, P.L., N.P., and U.F.), the European Respiratory Society (Long-Term Research Fellowship 675 to O.F.), and the Austrian, German and Swiss Pediatric Respiratory Society (Training Scholarship 2009 to O.F.).

\section{REFERENCES}

1. Braun-Fahrländer C, Gassner M, Grize L, et al. No further increase in asthma, hay fever and atopic sensitisation in adolescents living in Switzerland. Eur Respir J 2004;23:407-13.

2. Bush A, Menzies-Gow A. Phenotypic differences between pediatric and adult asthma. Proc Am Thorac Soc 2009;6:712-9.

3. Kuehni CE, Davis A, Brooke AM, Silverman M. Are all wheezing disorders in very young (preschool) children increasing in prevalence? Lancet 2001;357:1821-5.

4. Strunk RC, Szefler SJ, Phillips BR, et al. Relationship of exhaled nitric oxide to clinical and inflammatory markers of persistent asthma in children. J Allergy Clin Immunol 2003;112:883-92.

5. Syk J, Undén AL, Alving K. Relationship between exhaled nitric oxide and IgE sensitisation in patients with asthma: influence of steroid treatment. Clin Respir J 2009;3:143-51. 
6. Gratziou C, Lignos M, Dassiou M, Roussos C. Influence of atopy on exhaled nitric oxide in patients with stable asthma and rhinitis. Eur Respir J 1999;14:897-901.

7. Lúdvíksdóttir D, Janson C, Högman M, Hedenström H, Björnsson E, Boman G. Exhaled nitric oxide and its relationship to airway responsiveness and atopy in asthma. BHR-Study Group. Respir Med 1999;93:552-6.

8. Redington AE, Meng QH, Springall DR, et al. Increased expression of inducible nitric oxide synthase and cyclo-oxygenase- 2 in the airway epithelium of asthmatic subjects and regulation by corticosteroid treatment. Thorax 2001;56:351-7.

9. American Thoracic Society, European Respiratory Society. ATS/ERS recommendations for standardized procedures for the online and offline measurement of exhaled lower respiratory nitric oxide and nasal nitric oxide, 2005. Am J Respir Crit Care Med 2005;171:912-30.

10. Högman M, Meriläinen P. Extended NO analysis in asthma. J Breath Res 2007;1:024001.

11. Latzin P, Beck J, Griese M. Exhaled nitric oxide in healthy children: variability and a lack of correlation with atopy. Pediatr Allergy Immunol 2002;13:37-46.

12. Lundberg JO, Weitzberg E, Lundberg JM, Alving K. Nitric oxide in exhaled air. Eur Respir J 1996;9:2671-80.

13. Silkoff PE, McClean PA, Slutsky AS, et al. Marked flow-dependence of exhaled nitric oxide using a new technique to exclude nasal nitric oxide. Am J Respir Crit Care Med 1997;155:260-7.

14. Ito $\mathrm{Y}$, Adachi $\mathrm{Y}$, Itazawa $\mathrm{T}$, et al. Comparison of exhalation time methods ( $6 \mathrm{sec}$ vs. $10 \mathrm{sec})$ of a hand-held exhaled nitric oxide analyzer. Pediatr Pulmonol 2010;45:1005-8.

15. Napier E, Turner SW. Methodological issues related to exhaled nitric oxide measurement in children aged four to six years. Pediatr Pulmonol 2005;40:97-104.

16. Gangell CL, Hall GL, Stick SM, Sly PD; AREST CF. Lung function testing in preschool-aged children with cystic fibrosis in the clinical setting. Pediatr Pulmonol 2010;45:419-33.

17. Baraldi E, Dario C, Ongaro R, et al. Exhaled nitric oxide concentrations during treatment of wheezing exacerbation in infants and young children. Am J Respir Crit Care Med 1999;159:1284-8.

18. Buchvald F, Bisgaard H. FeNO measured at fixed exhalation flow rate during controlled tidal breathing in children from the age of $2 \mathrm{yr}$. Am J Respir Crit Care Med 2001;163:699-704.
19. Franklin PJ, Turner SW, Mutch RC, Stick SM. Measuring exhaled nitric oxide in infants during tidal breathing: methodological issues. Pediatr Pulmonol 2004;37:24-30.

20. Fuchs O, Latzin P, Thamrin C, et al. Normative data for lung function and exhaled nitric oxide in unsedated healthy infants. Eur Respir J 2011;37:1208-16.

21. Ratjen F, Kavuk I, Gärtig S, Wiesemann HG, Grasemann H. Airway nitric oxide in infants with acute wheezy bronchitis. Pediatr Allergy Immunol 2000;11:230-5.

22. Fuchs O, Latzin P, Kuehni CE, Frey U. Cohort Profile: the Bern Infant Lung Development Cohort. Int J Epidemiol 2011; e-pub ahead of print 13 January 2011.

23. Beydon N, Davis SD, Lombardi E, et al. An official American Thoracic Society/European Respiratory Society statement: pulmonary function testing in preschool children. Am J Respir Crit Care Med 2007;175: 1304-45.

24. Pijnenburg MWH. Exhaled nitric oxide in respiratory diseases other than asthma. In: Exhaled Biomarkers. Sheffield, UK: European Respiratory Society Journals, 2010:45-55.

25. Shaw DE, Wilson E, Pavord ID. Exhaled nitric oxide in asthma. In: Exhaled Biomarkers. Sheffield, UK: European Respiratory Society Journals, 2010:32-44.

26. Kalliola S, Malmberg P, Rito T, Pelkonen AS, Mäkelä MJ. Can we use portable nitric oxide analyzer in young children? Pediatr Pulmonol 2011;46:627-31.

27. Hadjikoumi I, Hassan A, Milner AD. Exhaled nitric oxide measurements in childhood asthma: comparison of two sampling techniques. Pediatr Res 2002;52:745-9.

28. Mahut B, Delacourt C, Zerah-Lancner F, De Blic J, Harf A, Delclaux C. Increase in alveolar nitric oxide in the presence of symptoms in childhood asthma. Chest 2004;125:1012-8.

29. Gabriele C, de Benedictis FM, de Jongste JC. Exhaled nitric oxide measurements in the first 2 years of life: methodological issues, clinical and epidemiological applications. Ital J Pediatr 2009;35:21.

30. Hall GL, Reinmann B, Wildhaber JH, Frey U. Tidal exhaled nitric oxide in healthy, unsedated newborn infants with prenatal tobacco exposure. J Appl Physiol 2002;92:59-66.

31. Bland JM, Altman DG. Statistical methods for assessing agreement between two methods of clinical measurement. Lancet 1986;1:307-10. 